\title{
Avaliação da qualidade de curso online empregando o QEOn: estudo de caso
}

\author{
Wagner Bandeira Andriola \\ Doutor em Filosofia e Ciências da Educação \\ Professor Titular da Universidade Federal do Ceará \\ Pesquisador do Conselho Nacional de Desenvolvimento Científico e Tecnológico \\ 凶andriola@yahoo.com
}

Recebido em 9 de junho de 2019

Aceito em 12 de setembro de 2019

\begin{abstract}
Resumo:
O estudo teve como objetivo avaliar a qualidade da formação do alunado de curso de graduação online em cinco dimensões propostas por H. W. Marsh em 1987: qualidade do tutor online; qualidade da interação tutor online com os alunos; qualidade da aprendizagem dos alunos; qualidade da relação tutor presencial com os alunos e qualidade dos processos avaliativos. Uma amostra de 31 alunos do curso Pedagogia online responderam ao Questionário para Avaliar a Qualidade de Ensino Online (QEOn). Os resultados demonstraram a elevada qualidade do curso de graduação online nos cinco atributos avaliados, conforme a percepção do alunado, o que proporciona elementos relevantes para a gestão acadêmica da Instituição de Ensino Superior (IES) tomar decisões com respeito aos demais cursos online.
\end{abstract}

Palavras-chave: Avaliação educacional, Ensino superior, Educação a distância.

\section{Evaluation of online course quality using the QEOn: case study}

\begin{abstract}
:
The aim of this study was to evaluate the quality of the online undergraduate course in five dimensions proposed by H. W. Marsh in 1987: quality of online tutor; quality of online tutor interaction with students; quality of student learning; quality of the tutor relationship with students; quality of evaluation processes. A sample of 31 students from the Online Pedagogy course answered the Questionnaire to Assess the Quality of Online Teaching (QEOn). The results showed the high quality of the online undergraduate course in the five attributes evaluated, according to the students' perception, which provides relevant elements for the academic management of the Higher Education Institution (IES) to make decisions regarding the other online courses.
\end{abstract}

Keywords: Educational evaluation, Higher education, Distance education. 


\title{
Evaluación de la calidad de curso online usando el QEOn: estudio de caso
}

\begin{abstract}
Resumen:
El estudio tuvo como objetivo evaluar la calidad de la formación del alumnado de curso de graduación online en cinco dimensiones propuestas por H. W. Marsh en 1987: calidad del tutor online; calidad de la interacción tutor online con los alumnos; calidad del aprendizaje de los alumnos; calidad de la relación tutor presencial con los alumnos; calidad de los procesos de evaluación. Una muestra de 31 alumnos del curso Pedagogía online respondieron al Cuestionario para Evaluar la Calidad de Enseñanza Online (QEOn). Los resultados demostraron la alta calidad del curso de graduación online en los cinco atributos evaluados, según la percepción del alumnado, lo que proporciona elementos relevantes para la gestión académica de la Institución de Enseñanza Superior (IES) tomar decisiones con respecto a los demás cursos online.

Palabras-clave: Evaluación educativa, Enseñanza superior, Educación online.
\end{abstract}

\section{AVALIAÇÃO EDUCACIONAL: BASES CONCEITUAIS}

A avaliação é uma atividade racional, científica e sistemática, que deve permitir a obtenção de novos conhecimentos acerca do objeto ou realidade avaliada, com o fito de poder-se planejar ações voltadas ao aprimoramento deste, consoante Andriola (1999). Com efeito, processos avaliativos conduzidos pelas Instituições de Ensino Superior (IES), denominados autoavaliações ou avaliações internas, se vinculam às funções de diagnóstico, planejamento, regulação e autorregulação, conforme Johnson e Glasman (1983), Andriola e Andriola (2009). Ademais, são obrigatórios para que a IES integre formalmente o Sistema de Educação Superior, cumprindo com as exigências concernentes a autorizações de funcionamento, credenciamento e recredenciamento (ANDRIOLA, 2005a; BRASIL, 2004). Portanto, a autoavaliação institucional é instrumento básico e obrigatório para todos os atos de regulação da IES, cujo exercício é prerrogativa do Estado, conforme a Lei nº 10.861, que criou o Sistema Nacional de Avaliação da Educação Superior (SINAES), segundo Andriola (2008b).

Sob este prisma, as IES passam a conhecer melhor a sua realidade organizacional e podem praticar atos regulatórios que considerem necessários para cumprir com maior qualidade e pertinência seus objetivos acadêmicos (ANDRIOLA; Mc DONALD, 2003). De acordo com Andriola (2005b), além de seus próprios estudos e pesquisas as IES também recebem as 
recomendações das Comissões de Avaliação Externa como feedback das ações executadas no decorrer do período analisado.

Dessa forma se revelam as funções mais importantes da autoavaliação institucional: produzir conhecimentos; diagnosticar o grau de consecução dos objetivos e finalidades essenciais da IES; identificar problemas e deficiências; aumentar a consciência pedagógica e a capacidade profissional da comunidade interna; tornar mais efetiva a vinculação da IES com o entorno social; julgar a relevância científica e social de suas atividades; prestar contas à sociedade (accountability); justificar publicamente sua existência (ANDRIOLA, 2004; LAVOR; ANDRIOLA; LIMA, 2015; ARAÚJO; ANDRIOLA, COELHO, 2018). Portanto, as ações de autoavaliação devem ser permanentes, constituindo-se numa cultura internalizada nos processos de planejamento, de gestão e de decisão institucionais (SANTORO TRIGUEIRO, 2004; BALZÁN, 1996; LIMA; ANDRIOLA, 2015; NUNES et al., 2015a; NUNES et al., 2015b).

A partir do exposto, o objetivo do artigo é apresentar as opiniões dos alunos do curso de graduação em Pedagogia online do Centro Universitário da Grande Fortaleza (UNIGrande), com o fito de demonstrar como o feedback dos alunos pode auxiliar na avaliação institucional e no planejamento estratégico, propiciando subsídios para o desenvolvimento de ações voltadas à melhoria de aspectos acadêmicos envolvidos na formação na modalidade de Educação a Distância (EaD). Para tanto, apresentar-se-ão informações acerca da avaliação da qualidade da EaD, através de estudos científicos.

\section{AVALIAÇÃO DA QUALIDADE DO ENSINO A DISTÂNCIA (EAD)}

Diferentemente dos países que optaram pela criação de Universidades exclusivamente para o Ensino a Distância (EaD), a partir da última década do século XX o Brasil provocou as universidades públicas a ofertarem os cursos presencias nessa nova modalidade. Para isto, deveriam repensar suas estruturas para a adoção da nova forma de ensino, revendo a organização administrativa e acadêmica, apropriando-a ao EaD. Assim, o Brasil começou efetivamente a ofertar cursos online somente a partir de 1996, com o advento da Lei de Diretrizes e Bases da Educação Nacional (LDB 9.394/96) que inseriu o EaD no sistema educacional, conforme o artigo $8^{\circ}$ (JESUS SILVA; RAMOS, 2011). Ademais, os avanços no campo 
da tecnologia, da comunicação e da informação afetaram drasticamente essa modalidade de cursos. Consoante Andriola e Loureiro (2005), vivemos o auge da Sociedade da Informação, o que implica numa forma distinta de organização econômica, social, política e cultural, resultando em modos inovadores de trabalhar, de comunicar-se, de relacionar-se, de aprender, de pensar e em última instância de viver.

Nesse contexto, as Tecnologias da Informação e da Comunicação (TIC) passaram a ocupar lugar ímpar na tarefa de difundir e ofertar o suporte necessário para um ensino de qualidade (LIMA; ANDRIOLA, 2013; TOLENTINO et al., 2013). Com o surgimento dos Learning Management System (LMS) houve incremento de ferramentas da internet aplicadas à aprendizagem, que motivou o surgimento de nomenclaturas tais como Ambientes Virtuais de Aprendizagem (AVA) ou entornos de aprendizagem on-line, e-learning e suas variações como aprendizagem híbrida ou blended-learning, aprendizagem móvel (MOORE, 1991; SILVA; LIMA; ANDRIOLA, 2016).

O impacto do uso das TIC nos cursos na modalidade EaD foi observado nos estudos abordando a qualidade da formação do alunado submetida a esse novo modelo pedagógico. Assim, diversos estudos internacionais (HOPE, 2001; EATON, 2002; MCKINNON et al., 2000; PHIPPS; MERISOTIS, 2000) e nacionais (RAPCHAN et al., 2002; ANDRIOLA; LOUREIRO, 2005; CASAGRANDE et al., 2008; TOLENTINO et al., 2013; TEIXEIRA et al., 2014; SALES et al. 2011; SILVA et al., 2015; RIBEIRO et al. 2018) têm discutido a qualidade de cursos online. Essas pesquisas geralmente baseiam-se no emprego de instrumentos para avaliar os cursos online considerando-se diferentes aspectos inerentes à qualidade, consoante Moore (1991), Maia e Mattar (2007), Bastos e Rovaris (2016). Sob essa ótica, empregou-se o instrumento de Avaliação da Qualidade de Ensino Online (QEOn) para se obter indícios da qualidade da formação resultante de um curso de Pedagogia online.

Ressalte-se que o QEOn baseia-se na metodologia SET (do inglês Students' Evaluation of Teaching) para avaliar a efetividade do ensino sob a ótica discente, proposto por H. W. Marsh (1987), posteriormente revisado por Marsch e Bailey (1993). Convém salientar que, no Brasil, Silva et al. (2015) efetivaram estudo voltado à validação do SET e encontraram resultados muito promissores no que tange ao uso do instrumento para o diagnóstico da qualidade dos cursos. Embora o SET avalie aspectos relacionados à qualidade dos cursos, ele não se aplica integralmente à realidade do ensino semipresencial do UNIGrande. Assim sendo, houve a 
necessidade de desenvolver-se outro instrumento para avaliar os cursos de EaD sob a perspectiva de cinco atributos reveladores da qualidade do ensino online, a saber: qualidade do tutor online; qualidade da interação tutor online com os alunos; qualidade da aprendizagem dos alunos; qualidade da relação tutor presencial com os alunos; qualidade dos processos avaliativos.

Baseado nesses atributos, Silva et al. (2017) desenvolveu um instrumental, através de estudo com amostra de 417 alunos que participaram de cursos EaD promovidos pela Universidade Federal do Ceará (UFC). Os resultados obtidos indicaram elevada qualidade métrica do novo instrumental, no que tange à validade fatorial (os resultados obtidos explicaram 69\% da variância total através de cinco componentes extraídas) e à consistência interna (os coeficientes alfa de Cronbach variaram de 0,82 a 0,94 nas cinco componentes extraídas), que foi posteriormente denominado de Questionário para Avaliar a Qualidade do Ensino Online (QEOn).

A partir das dimensões avaliadas pelo QEOn pode-se gerar indicadores para averiguar a qualidade do ensino online, propícios ao fornecimento de informações para a gestão acadêmica e a tomada de decisões, consoante Andriola e Loureiro (2005), Vasconselos et al. (2011), Sales et al. (211) e Ribeiro et al. (2018). No Quadro 1, a seguir, são apresentadas as especificações dos cinco indicadores do QEOn, empregados no diagnóstico da qualidade de cursos online.

Quadro 1 - Indicadores para avaliar a qualidade do ensino online componentes do QEOn

\begin{tabular}{||l|l||}
\hline \multicolumn{1}{||}{ Indicadores } & \multicolumn{1}{c|}{ Atributos Idealizados } \\
\hline \hline Tutor online & $\begin{array}{l}\text { Este indicador está diretamente relacionado com a qualidade da tutoria online. Se o tutor online } \\
\text { apresentar dinamismo, entusiasmo, não se limitar ao conteúdo disponível na plataforma, estiver } \\
\text { atento ao aprendizado do aluno, apresentar o conteúdo de forma satisfatória, relacionar teoria } \\
\text { e prática, certamente ele terá um bom escore nesse indicador. }\end{array}$ \\
\hline $\begin{array}{l}\text { Interação tutor } \\
\text { Online/Aluno }\end{array}$ & $\begin{array}{l}\text { Este indicador mede o quão estimulador é o tutor online nas discussões dos fóruns. Alguns } \\
\text { pesquisadores defendem a interação tutor online-aluno como sendo um dos fatores } \\
\text { fundamentais para o sucesso do ensino online. }\end{array}$ \\
\hline $\begin{array}{l}\text { Aprendizagem } \\
\text { Tutor }\end{array}$ & $\begin{array}{l}\text { O nível de aprendizagem adquirida pelo aluno é medido por esse indicador. O próprio aluno } \\
\text { valora sua aprendizagem ao longo do curso, e esse indicador pode ser considerado como sendo } \\
\text { como um profissional de suporte presente nos polos de apoio presencial. Este indicador mede a } \\
\text { relação entre o tutor presencial e o aluno. }\end{array}$ \\
\hline $\begin{array}{l}\text { Presencial/Aluno de autoavaliação. } \\
\text { Torna-se necessário sistemáticas de avaliação condizentes com a modalidade a } \\
\text { Avaliativos }\end{array}$ & \begin{tabular}{l} 
Este indicador está relacionado à adequação dos processos avaliativos ao curso online. \\
\hline Processos
\end{tabular} \\
\hline
\end{tabular}

Fonte: Silva et al. (2017). 
Para a geração dos cinco indicadores relacionados à qualidade do ensino online, conforme proposto por Silva et al. (2017), aplicou-se o QEOn em uma amostra de 31 alunos regularmente matriculados no curso de Pedagogia online do Centro Universitário da Grande Fortaleza (UNIGrande) no período letivo de 2018.2. Convém destacar que além destes cinco indicadores do QEOn obtiveram-se informações acerca do perfil do curso de Pedagogia online e da qualidade dos materiais pedagógicos.

\section{PROCEDIMENTOS METODOLÓGICOS}

Trata-se de um estudo ex post-facto, de natureza descritiva, baseado em dados qualiquantitativos obtidos junto a uma amostra de alunos regularmente matriculados no curso de Pedagogia online do UNIGrande.

\section{Amostra}

A amostra foi composta por 31 alunos regularmente matriculados no curso de Pedagogia online do UNIGrande, cuja amplitude de idade variou de 22 a 35 anos, com média de 23,5 anos ( $d p=2,5$ anos) e moda de 30 anos ( $n=12$ ou 38,7\%). A maioria desses alunos ( $n=$ 17 ou 54,8\%) concluiu o Ensino Médio em escolas públicas, com a minoria tendo concluído em escolas particulares ( $\mathrm{n}=14$ ou $45,2 \%)$.

\section{Instrumento}

Empregou-se o Questionário para Avaliar a Qualidade do Ensino Online (QEOn), que é composto por 29 itens que se relacionam a cinco atributos de qualidade do ensino online, a saber: qualidade do tutor online; qualidade da interação tutor online com os cursistas; qualidade da aprendizagem dos cursistas; qualidade da relação tutor- presencial com os cursistas; qualidade dos processos avaliativos. A escala de resposta do QEOn é do tipo Likert, com quatro pontos de gradação: 1 - Concordo Totalmente; 2 - Concordo; 3 - Discordo; 4 - Discordo Totalmente. 


\section{Procedimento}

O QEOn foi aplicado aos alunos regularmente matriculados no curso de Pedagogia online do Centro Universitário da Grande Fortaleza (UNIGrande) no período letivo 2018.2, que estavam a participar de uma tutoria presencial, após ter-se explicado os propósitos do estudo e solicitado formalmente o aceite em preencher o referido instrumento. Os alunos preencheram o Termo de Consentimento Livre e Esclarecido (TLEC), conforme as normas adotadas pelo Comitê de Ética da IES.

\section{APRESENTAÇÃO E DISCUSSÃO DOS RESULTADOS}

Os principais resultados são apresentados a seguir, organizados em sete tópicos, estando cinco destes em conformidade com os indicadores do QEOn: i) o perfil do curso de Pedagogia online; ii) a qualidade do tutor online; iii) a qualidade da interação do tutor online com os alunos; iv) a qualidade da interação do tutor presencial com os alunos; v) a qualidade dos materiais pedagógicos; vi) a qualidade da aprendizagem dos alunos; vii) a qualidade dos processos avaliativos.

\section{Perfil do Curso de Pedagogia online}

Indagou-se aos alunos o quão desafiador e estimulador era o curso de Pedagogia online, conforme os dados da Tabela 1, a seguir apresentada.

Tabela 1 - O quão desafiador e estimulante é o curso

\begin{tabular}{ccccc}
\hline $\begin{array}{c}\text { CATEGORIAS DE } \\
\text { RESPOSTAS }\end{array}$ & FREQUÊNCIA & PORCENTUAL & $\begin{array}{c}\text { PORCENTAGEM } \\
\text { VÁLIDA }\end{array}$ & $\begin{array}{c}\text { PORCENTAGEM } \\
\text { ACUMULATIVA }\end{array}$ \\
\hline Concordo & 25 & 80,6 & 80,6 & 80,6 \\
Concordo totalmente & 6 & 19,4 & 19,4 & 100,0 \\
Total & 31 & 100,0 & 100,0 & \\
\hline
\end{tabular}

Fonte: Pesquisa direta (2018). 
Consoante os dados, todos os alunos opinaram que o curso de Pedagogia online é desafiador e estimulante, ajustando-se às premissas teóricas de Moore (1991), para quem um curso deve converter-se em uma experiência desafiadora para o alunado, ademais de estimuladora, para que estes busquem novos aprendizados durante a formação. Posteriormente, indagou-se acerca da qualidade e pertinência dos conteúdos abordados durante a formação, conforme os dados da Tabela 2.

Tabela 2 - Qualidade e pertinência dos conteúdos abordados durante a formação

\begin{tabular}{ccccc}
\hline $\begin{array}{c}\text { CATEGORIAS DE } \\
\text { RESPOSTAS }\end{array}$ & FREQUÊNCIA & PORCENTUAL & $\begin{array}{c}\text { PORCENTAGEM } \\
\text { VÁLIDA }\end{array}$ & $\begin{array}{c}\text { PORCENTAGEM } \\
\text { ACUMULATIVA }\end{array}$ \\
\hline Concordo & 19 & 61,3 & 61,3 & 61,3 \\
Concordo totalmente & 11 & 35,5 & 35,5 & 96,8 \\
Discordo totalmente & 1 & 3,2 & 3,2 & 100,0 \\
Total & $\mathbf{3 1}$ & $\mathbf{1 0 0 , 0}$ & $\mathbf{1 0 0 , 0}$ & \\
\hline
\end{tabular}

Fonte: Pesquisa direta (2018).

A expressiva maioria dos alunos opinou que aprendeu algo pertinente no curso de Pedagogia online ( $n=30$ ou 96,8\%), embora tenha havido discordância ( $n=1$ ou 3,2\%). Tal padrão de resposta corrobora com os achados de Scherer e Brito (2014), para quem os cursos online devem proporcionar experiências úteis aos alunos, através de conteúdos componentes de um currículo rico e versátil às exigências da sociedade e do mercado de trabalho.

A seguir apresenta-se informação acerca do incremento do interesse do alunado pelas temáticas curriculares, consoante os dados da Tabela 3.

Tabela 3 - Incremento do interesse sobre as temáticas curriculares como consequência do curso

\begin{tabular}{ccccc}
\hline $\begin{array}{c}\text { CATEGORIAS DE } \\
\text { RESPOSTAS }\end{array}$ & FREQUÊNCIA & PORCENTUAL & $\begin{array}{c}\text { PORCENTAGEM } \\
\text { VÁLIDA }\end{array}$ & $\begin{array}{c}\text { PORCENTAGEM } \\
\text { ACUMULATIVA }\end{array}$ \\
\hline Concordo & 28 & 90,3 & 90,3 & 90,3 \\
Concordo totalmente & 3 & 9,7 & 9,7 & 100,0 \\
Total & 31 & $\mathbf{1 0 0 , 0}$ & $\mathbf{1 0 0 , 0}$ & \\
\hline
\end{tabular}

Fonte: Pesquisa direta (2018). 
Os dados revelam que todos os alunos opinaram que houve incremento no interesse pelas temáticas abordadas no decorrer da formação, ajustando-se às premissas teóricas de Garridson (1993) e Moore (1991), para quem um curso deve ser estimulador e indutor de novos aprendizados ao longo do processo de formação.

Os alunos foram sondados acerca da aderência dos conteúdos pedagógicos aos objetivos propostos para o curso, conforme as informações da Tabela 4.

Tabela 4 - Os objetivos propostos estão de acordo com o que foi ensinado durante o curso

\begin{tabular}{ccccc}
\hline $\begin{array}{c}\text { CATEGORIAS DE } \\
\text { RESPOSTAS }\end{array}$ & FREQUÊNCIA & PORCENTUAL & $\begin{array}{c}\text { PORCENTAGEM } \\
\text { VÁLIDA }\end{array}$ & $\begin{array}{c}\text { PORCENTAGEM } \\
\text { ACUMULATIVA }\end{array}$ \\
\hline Concordo & 19 & 61,3 & 61,3 & 61,3 \\
Concordo totalmente & 11 & 35,5 & 35,5 & 96,8 \\
Discordo & 1 & 3,2 & 3,2 & 100,0 \\
Total & $\mathbf{3 1}$ & $\mathbf{1 0 0 , 0}$ & $\mathbf{1 0 0 , 0}$ & \\
\hline
\end{tabular}

Fonte: Pesquisa direta (2018).

De acordo com a opinião da expressiva maioria do alunado ( $n=30$ ou $96,8 \%$ ) os conteúdos pedagógicos abordados durante a formação são aderentes aos objetivos propostos para o curso, ajustando-se às ideias de Moore e Kearsley (2008), para quem as atividades de formação devem estar aderentes aos objetivos propostos para o curso.

QUanto à qualidade do Ambiente Virtual de Aprendizagem (AVA), os alunos foram indagados acerca da contribuição das leituras complementares para incrementar a compreensão dos conteúdos programáticos, conforme os dados da Tabela 5.

Tabela 5 - Contribuição das leituras complementares, chat, fóruns, portfólios para a compreensão dos conteúdos programáticos

\begin{tabular}{ccccc}
\hline $\begin{array}{c}\text { CATEGORIAS DE } \\
\text { RESPOSTAS }\end{array}$ & FREQUÊNCIA & PORCENTUAL & $\begin{array}{c}\text { PORCENTAGEM } \\
\text { VÁLIDA }\end{array}$ & PORCENTAGEM ACUMULATIVA \\
\hline Concordo & 23 & 74,2 & 74,2 & 74,2 \\
Concordo totalmente & 7 & 22,6 & 22,6 & 96,8 \\
Discordo & 1 & 3,2 & 3,2 & 100,0 \\
Total & $\mathbf{3 1}$ & $\mathbf{1 0 0 , 0}$ & $\mathbf{1 0 0 , 0}$ & \\
\hline
\end{tabular}

Fonte: Pesquisa direta (2018). 
Observa-se que a expressiva maioria do alunado reconheceu que houve relevante contribuição das leituras complementares e de outras estratégias de indução à interação discente para a compreensão dos conteúdos programáticos abordados pelos tutores $(\mathrm{n}=30$ ou 96,8\%), consoante a opinião de Garridson (1993).

Posteriormente os alunos foram indagados acerca da acessibilidade metodológica, instrumental e comunicacional do Ambiente Virtual de Aprendizagem, conforme os dados da Tabela 6.

Tabela 6 - Nível de acessibilidade metodológica, instrumental e comunicacional do Ambiente Virtual de Aprendizagem (AVA)

\begin{tabular}{ccccc}
\hline $\begin{array}{c}\text { CATEGORIAS DE } \\
\text { RESPOSTAS }\end{array}$ & FREQUÊNCIA & PORCENTUAL & $\begin{array}{c}\text { PORCENTAGEM } \\
\text { VÁLIDA }\end{array}$ & PORCENTAGEM ACUMULATIVA \\
\hline Concordo & 22 & 71,0 & 71,0 & 71,0 \\
Concordo totalmente & 7 & 22,6 & 22,6 & 93,5 \\
Discordo & 2 & 6,5 & 6,5 & 100,0 \\
Total & 31 & $\mathbf{1 0 0 , 0}$ & $\mathbf{1 0 0 , 0}$ & \\
\hline
\end{tabular}

Fonte: Pesquisa direta (2018).

Consoante os dados, observa-se que a expressiva maioria do alunado reconheceu que o Ambiente Virtual de Aprendizagem (AVA) permite a acessibilidade metodológica, instrumental e comunicacional, facilitando a compreensão dos conteúdos programáticos abordados pelos tutores ( $\mathrm{n}=29$ ou 93,5\%).

\section{Qualidade do Tutor online}

O alunado foi sondado acerca da qualidade da tutoria online, inicialmente indagandose acerca do entusiasmo do tutor, conforme a Tabela 7. 
Tabela 7 - Entusiasmo demonstrado pelo tutor online ao ministrar o curso

\begin{tabular}{ccccc}
\hline $\begin{array}{c}\text { CATEGORIAS DE } \\
\text { RESPOSTAS }\end{array}$ & FREQUÊNCIA & PORCENTUAL & $\begin{array}{c}\text { PORCENTAGEM } \\
\text { VÁLIDA }\end{array}$ & $\begin{array}{c}\text { PORCENTAGEM } \\
\text { ACUMULATIVA }\end{array}$ \\
\hline Concordo & 16 & 51,6 & 51,6 & 51,6 \\
Concordo totalmente & 13 & 41,9 & 41,9 & 93,5 \\
Discordo & 2 & 6,5 & 6,5 & 100,0 \\
Total & \multirow{31}{*}{$\mathbf{1 0 0 , 0}$} & $\mathbf{1 0 0 , 0}$ & \\
\hline
\end{tabular}

Fonte: Pesquisa direta (2018).

Conforme a opinião da expressiva maioria do alunado ( $n=29$ ou $93,5 \%)$ o tutor online demonstrou entusiasmo ao ministrar o curso, ajustando-se às ideias formuladas por Bleicher (2015) e Peters (2003), para quem um curso deve conter tutores motivados e entusiasmados com as ações de formação discente.

Mais adiante, o alunado foi indagado acerca de o tutor sugerir a consulta de sites e vídeos para incrementar a qualidade do aprendizado, conforme a Tabela 8.

Tabela 8 - O tutor online faz sugestões a consultas de sites e vídeos para incrementar a qualidade do aprendizado

\begin{tabular}{ccccc}
\hline $\begin{array}{c}\text { CATEGORIAS DE } \\
\text { RESPOSTAS }\end{array}$ & FREQUÊNCIA & PORCENTUAL & $\begin{array}{c}\text { PORCENTAGEM } \\
\text { VÁLIDA }\end{array}$ & PORCENTAGEM ACUMULATIVA \\
\hline Concordo & 20 & 64,5 & 64,5 & 64,5 \\
Concordo totalmente & 10 & 32,3 & 32,3 & 96,8 \\
Discordo & 1 & 3,2 & 3,2 & 100,0 \\
Total & $\mathbf{3 1}$ & $\mathbf{1 0 0 , 0}$ & $\mathbf{1 0 0 , 0}$ & \\
\hline
\end{tabular}

Fonte: Pesquisa direta (2018).

Com base na opinião da expressiva maioria do alunado ( $n=30$ ou $96,8 \%$ ) o tutor online faz sugestões para que os alunos consultem sites e vídeos, de modo a incrementar a qualidade do aprendizado. Este posicionamento ajusta-se às ideias apregoadas por Bleicher (2015), Moore e Kearsley (2008), para quem o tutor deve possibilitar aos alunos novas maneiras de adensar o aprendizado.

No que tange à qualidade da relação tutor online-alunado, a Tabela 9 apresenta-nos informações acerca do quão amigável foi o relacionamento com a turma. 
Tabela 9 - O tutor online foi amigável na relação com os cursistas

\begin{tabular}{ccccc}
\hline $\begin{array}{c}\text { CATEGORIAS DE } \\
\text { RESPOSTAS }\end{array}$ & FREQUÊNCIA & PORCENTUAL & $\begin{array}{c}\text { PORCENTAGEM } \\
\text { VÁLIDA }\end{array}$ & $\begin{array}{c}\text { PORCENTAGEM } \\
\text { ACUMULATIVA }\end{array}$ \\
\hline Concordo & 23 & 74,2 & 74,2 & 74,2 \\
Concordo totalmente & 1 & 3,2 & 3,2 & 77,4 \\
Discordo & 7 & 22,6 & 22,6 & 100,0 \\
Total & 31 & $\mathbf{1 0 0 , 0}$ & $\mathbf{1 0 0 , 0}$ & \\
\hline
\end{tabular}

Fonte: Pesquisa direta (2018).

Conforme a expressiva maioria do alunado $(n=24$ ou 77,4\%) o tutor online estabeleceu relacionamento amigável, aspecto ressaltado por Peters (2003) como algo desejável para as situações cotidianas de formação de discentes na modalidade EaD.

No que concerne ao emprego rotineiro de resultados de pesquisa nos processos formativos por parte do tutor online, a Tabela 10 apresenta-nos informações relevantes.

Tabela 10 - O tutor online comenta pesquisas desenvolvidas na área de estudo

\begin{tabular}{ccccc}
\hline $\begin{array}{c}\text { CATEGORIAS DE } \\
\text { RESPOSTAS }\end{array}$ & FREQUÊNCIA & PORCENTUAL & $\begin{array}{c}\text { PORCENTAGEM } \\
\text { VÁLIDA }\end{array}$ & $\begin{array}{c}\text { PORCENTAGEM } \\
\text { ACUMULATIVA }\end{array}$ \\
\hline Concordo & 21 & 67,7 & 67,7 & 67,7 \\
Concordo totalmente & 1 & 3,2 & 3,2 & 71,0 \\
Discordo & 9 & 29,0 & 29,0 & 100,0 \\
Total & 31 & $\mathbf{1 0 0 , 0}$ & $\mathbf{1 0 0 , 0}$ & \\
\hline
\end{tabular}

Fonte: Pesquisa direta (2018).

Segundo a maioria do alunado ( $\mathrm{n}=22$ ou 71\%) o tutor online empregou resultados de pesquisa nos processos formativos, característica ressaltada por Garridson (1993) como algo vital à qualidade do aprendizado dos alunos.

Acerca do emprego pelo tutor online de material complementar para a promoção e o incremento do aprendizado do alunado, a Tabela 11 apresenta-nos dados relevantes. 
Tabela 11 - O tutor online propôs leituras complementares que facilitaram o aprendizado

\begin{tabular}{ccccc}
\hline $\begin{array}{c}\text { CATEGORIAS DE } \\
\text { RESPOSTAS }\end{array}$ & FREQUÊNCIA & PORCENTUAL & $\begin{array}{c}\text { PORCENTAGEM } \\
\text { VÁLIDA }\end{array}$ & $\begin{array}{c}\text { PORCENTAGEM } \\
\text { ACUMULATIVA }\end{array}$ \\
\hline Concordo & 25 & 80,6 & 80,6 & 80,6 \\
Concordo totalmente & 6 & 19,4 & 19,4 & 100,0 \\
Total & 31 & 100,0 & 100,0 & \\
\hline
\end{tabular}

Fonte: Pesquisa direta (2018).

De acordo com a totalidade do alunado, o tutor online propôs leituras complementares que facilitaram o aprendizado discente, aspecto extremamente positivo ao processo formativo em cursos online, conforme Garridson (1993) e Peters (2003).

\section{Qualidade da Interação do Tutor online com os Alunos}

O alunado também foi sondado acerca da qualidade da tutoria online, através da capacidade de elucidar indagações e dúvidas, conforme a Tabela 12.

Tabela 12 - O tutor online rotineiramente elucida as indagações dos cursistas?

\begin{tabular}{ccccc}
\hline $\begin{array}{c}\text { CATEGORIAS DE } \\
\text { RESPOSTAS }\end{array}$ & FREQUÊNCIA & PORCENTUAL & $\begin{array}{c}\text { PORCENTAGEM } \\
\text { VÁLIDA }\end{array}$ & $\begin{array}{c}\text { PORCENTAGEM } \\
\text { ACUMULATIVA }\end{array}$ \\
\hline Concordo & 17 & 54,8 & 54,8 & 54,8 \\
Concordo totalmente & 13 & 41,9 & 41,9 & 96,8 \\
Discordo & 1 & 3,2 & 3,2 & 100,0 \\
Total & 31 & $\mathbf{1 0 0 , 0}$ & $\mathbf{1 0 0 , 0}$ & \\
\hline
\end{tabular}

Fonte: Pesquisa direta (2018).

Consoante a expressiva maioria do alunado ( $\mathrm{n}=30$ ou 96,8\%), o tutor online expressa de modo rotineiro capacidade de elucidar indagações e dúvidas, característica vital à formação e ao aprendizado dos alunos na modalidade EaD.

Averiguou-se a capacidade do tutor online relacionar as implicações do conteúdo abordado, confrontando-o com outras teorias, conforme a Tabela 13. 
Tabela 13 - O tutor relaciona as implicações do conteúdo abordado com outras teorias acerca do tema

\begin{tabular}{ccccc}
\hline $\begin{array}{c}\text { CATEGORIAS DE } \\
\text { RESPOSTAS }\end{array}$ & FREQUÊNCIA & PORCENTUAL & $\begin{array}{c}\text { PORCENTAGEM } \\
\text { VÁLIDA }\end{array}$ & $\begin{array}{c}\text { PORCENTAGEM } \\
\text { ACUMULATIVA }\end{array}$ \\
\hline Concordo & 24 & 77,4 & 77,4 & 77,4 \\
Concordo totalmente & 5 & 16,1 & 16,1 & 93,5 \\
Discordo & 2 & 6,5 & 6,5 & 100,0 \\
Total & 31 & $\mathbf{1 0 0 , 0}$ & $\mathbf{1 0 0 , 0}$ & \\
\hline
\end{tabular}

Fonte: Pesquisa direta (2018).

Conforme a expressiva maioria do alunado ( $\mathrm{n}=29$ ou 93,5\%) o tutor presencial relaciona as implicações do conteúdo abordado, confrontando-o com outras teorias, característica desejável para despertar o pensamento crítico do alunado em formação, consoante Andriola (2001).

Posteriormente, avaliou-se a capacidade do tutor presencial apresentar os fundamentos teóricos das atividades virtuais desenvolvidas, conforme a Tabela 14.

Tabela 14 - O tutor apresenta os fundamentos das atividades virtuais desenvolvidas

\begin{tabular}{ccccc}
\hline $\begin{array}{c}\text { CATEGORIAS DE } \\
\text { RESPOSTAS }\end{array}$ & FREQUÊNCIA & PORCENTUAL & $\begin{array}{c}\text { PORCENTAGEM } \\
\text { VÁLIDA }\end{array}$ & $\begin{array}{c}\text { PORCENTAGEM } \\
\text { ACUMULATIVA }\end{array}$ \\
\hline Concordo & 25 & 80,6 & 80,6 & 80,6 \\
Concordo totalmente & 1 & 3,2 & 3,2 & 83,9 \\
Discordo & 5 & 16,1 & 16,1 & 100,0 \\
Total & 31 & $\mathbf{1 0 0 , 0}$ & $\mathbf{1 0 0 , 0}$ & \\
\hline
\end{tabular}

Fonte: Pesquisa direta (2018).

De acordo com a expressiva maioria do alunado ( $\mathrm{n}=26$ ou 83,9\%) o tutor apresentou os fundamentos das atividades virtuais desenvolvidas no decorrer das ações de formação, característica desejável para despertar o pensamento científico. 


\section{Qualidade da Interação do Tutor Presencial com os Alunos}

O alunado também foi sondado acerca da qualidade da tutoria presencial, inicialmente indagando-se acerca da capacidade de elucidar indagações e dúvidas, conforme as informações da Tabela 15.

Tabela 15 - O tutor presencial rotineiramente elucida as indagações dos cursistas?

\begin{tabular}{ccccc}
\hline CATEGORIAS DE RESPOSTAS FREQUÊNCIA & PORCENTUAL & PORCENTAGEM VÁLIDA & PORCENTAGEM ACUMULATIVA \\
\hline Concordo & 24 & 77,4 & 77,4 & 77,4 \\
Concordo totalmente & 5 & 16,1 & 16,1 & 93,5 \\
Discordo & 2 & 6,5 & 6,5 & 100,0 \\
Total & 31 & $\mathbf{1 0 0 , 0}$ & $\mathbf{1 0 0 , 0}$ & \\
\hline
\end{tabular}

Fonte: Pesquisa direta (2018).

De acordo com a maioria do alunado ( $n=29$ ou 93,5\%), o tutor presencial expressa, de modo rotineiro, capacidade de elucidar indagações e dúvidas, característica vital à formação e ao aprendizado dos alunos na modalidade EaD.

Posteriormente, avaliou-se a capacidade do tutor presencial apresentar os fundamentos teóricos das atividades virtuais desenvolvidas, conforme a Tabela 16.

Tabela 16 - O tutor presencial apresenta os fundamentos das atividades virtuais desenvolvidas

\begin{tabular}{ccccc}
\hline $\begin{array}{c}\text { CATEGORIAS DE } \\
\text { RESPOSTAS }\end{array}$ & FREQUÊNCIA & PORCENTUAL & $\begin{array}{c}\text { PORCENTAGEM } \\
\text { VÁLIDA }\end{array}$ & $\begin{array}{c}\text { PORCENTAGEM } \\
\text { ACUMULATIVA }\end{array}$ \\
\hline Concordo & 22 & 71,0 & 71,0 & 71,0 \\
Concordo totalmente & 6 & 19,4 & 19,4 & 90,3 \\
Discordo & 3 & 9,7 & 9,7 & 100,0 \\
Total & 31 & $\mathbf{1 0 0 , 0}$ & $\mathbf{1 0 0 , 0}$ & \\
\hline
\end{tabular}

Fonte: Pesquisa direta (2018).

Consoante a expressiva maioria do alunado ( $n=28$ ou 90,3\%) o tutor presencial apresentou os fundamentos das atividades virtuais desenvolvidas no decorrer das ações de formação, característica desejável para despertar o pensamento científico. Para finalizar, 
avaliou-se a capacidade da equipe de tutoria para realizar as atividades virtuais, em termos de conhecimentos, habilidades e atitudes, conforme as informações da Tabela 17.

Tabela 17 - Adequação dos conhecimentos, habilidades e atitudes da equipe de tutoria para a realização das atividades de formação

\begin{tabular}{ccccc}
\hline $\begin{array}{c}\text { CATEGORIAS DE } \\
\text { RESPOSTAS }\end{array}$ & FREQUÊNCIA & PORCENTUAL & $\begin{array}{c}\text { PORCENTAGEM } \\
\text { VÁLIDA }\end{array}$ & PORCENTAGEM ACUMULATIVA \\
\hline Concordo & 22 & 71,0 & 71,0 & 71,0 \\
Concordo totalmente & 6 & 19,4 & 19,4 & 90,3 \\
Discordo & 3 & 9,7 & 9,7 & 100,0 \\
Total & $\mathbf{3 1}$ & $\mathbf{1 0 0 , 0}$ & $\mathbf{1 0 0 , 0}$ & \\
\hline
\end{tabular}

Fonte: Pesquisa direta (2018).

Consoante a expressiva maioria do alunado $(n=28$ ou 90,3\%) a equipe de tutoria demonstrou capacidade, conhecimentos, habilidades e atitudes, para realizar as atividades virtuais intrínsecas ao modelo de formação de alunos via EaD, ajustando-se ao preceituado por Silva et al. (2015), Scherer e Brito (2014).

\section{Qualidade dos Materiais Pedagógicos}

A qualidade do material pedagógico empregado na formação de alunos é um aspecto por demais relevante no âmbito educacional, consoante Bleicher (2015), Albuquerque e Silva (2012), Silva et al. (2015), Garridson (1993), Scherer e Brito (2014), Peters (2003), Moore e Kearsley (2008). Sob este prisma, avaliou-se a opinião do alunado acerca da qualidade do material pedagógico utilizado rotineiramente durante a formação, conforme os dados presentes na Tabela 18.

Tabela 18 - Qualidade do material pedagógico empregado no curso

\begin{tabular}{ccccc}
\hline $\begin{array}{c}\text { CATEGORIAS DE } \\
\text { RESPOSTAS }\end{array}$ & FREQUÊNCIA & PORCENTUAL & $\begin{array}{c}\text { PORCENTAGEM } \\
\text { VÁLIDA }\end{array}$ & $\begin{array}{c}\text { PORCENTAGEM } \\
\text { ACUMULATIVA }\end{array}$ \\
\hline Concordo & 15 & 48,4 & 48,4 & 48,4 \\
Concordo totalmente & 6 & 19,4 & 19,4 & 67,7 \\
Discordo & 9 & 29,0 & 29,0 & 96,8 \\
Discordo totalmente & 1 & 3,2 & 3,2 & 100,0 \\
Total & $\mathbf{3 1}$ & $\mathbf{1 0 0 , 0}$ & $\mathbf{1 0 0 , 0}$ & \\
\hline
\end{tabular}


Fonte: Pesquisa direta (2018).

A maioria do alunado $(n=21$ ou 67,7\%) reconheceu a qualidade do material pedagógico empregado nas ações de formação. Não obstante, houve um grupo minoritário ( $\mathrm{n}=10$ ou 32,3\%) que não reconheceu a qualidade do material pedagógico da formação. Esse é um aspecto que deve preocupar sobremaneira a equipe de gestão pedagógica do UNIGrande, pois o material pedagógico é um insumo diretamente vinculado à qualidade do aprendizado do alunado, conforme Bleicher (2015), Albuquerque e Silva (2012), Silva et al. (2015), Garridson (1993), Scherer e Brito (2014), Peters (2003), Moore e Kearsley (2008).

Posteriormente, avaliou-se a opinião do alunado acerca da disponibilidade de textos para leitura, conforme os dados da Tabela 19.

Tabela 19 - O curso requer a leitura de textos disponibilizados

\begin{tabular}{ccccc}
\hline $\begin{array}{c}\text { CATEGORIAS DE } \\
\text { RESPOSTAS }\end{array}$ & FREQUÊNCIA & PORCENTUAL & $\begin{array}{c}\text { PORCENTAGEM } \\
\text { VÁLIDA }\end{array}$ & $\begin{array}{c}\text { PORCENTAGEM } \\
\text { ACUMULATIVA }\end{array}$ \\
\hline Concordo & 25 & 80,6 & 80,6 & 80,6 \\
Concordo totalmente & 5 & 16,1 & 16,1 & 96,8 \\
Discordo & 1 & 3,2 & 3,2 & 100,0 \\
Total & 31 & $\mathbf{1 0 0 , 0}$ & $\mathbf{1 0 0 , 0}$ & \\
\hline
\end{tabular}

Fonte: Pesquisa direta (2018).

A expressiva maioria do alunado ( $\mathrm{n}=30$ ou 96,8\%) reconheceu que houve a disponibilização de material pedagógico (textos) empregado nas ações de formação, dos quais se exigiam a leitura. Trata-se de uma estratégia que deve ser garantida pela equipe de gestão pedagógica do curso, pois o material pedagógico é insumo diretamente vinculado à qualidade do aprendizado, conforme Preti (2000) e Peters (2003).

\section{Qualidade da Aprendizagem dos Alunos}

A qualidade da aprendizagem é o principal indicador acerca da consistência, fortaleza, eficiência e eficácia da formação educacional, consoante Bleicher (2015), Albuquerque e Silva (2012), Silva et al. (2015), Garridson (1993), Scherer e Brito (2014), Peters (2003), Moore e Kearsley (2008). 
Assim, avaliou-se a opinião do alunado acerca da qualidade do aprendizado no decorrer do processo de formação ao qual foi submetido, focando sobre a indução a participar das discussões via fórum, conforme revelam os dados da Tabela 20.

Tabela 20 - Os alunos foram encorajados a participar das discussões no fórum

\begin{tabular}{ccccc}
\hline $\begin{array}{c}\text { CATEGORIAS DE } \\
\text { RESPOSTAS }\end{array}$ & FREQUÊNCIA & PORCENTUAL & $\begin{array}{c}\text { PORCENTAGEM } \\
\text { VÁLIDA }\end{array}$ & $\begin{array}{c}\text { PORCENTAGEM } \\
\text { ACUMULATIVA }\end{array}$ \\
\hline Concordo & 15 & 48,4 & 48,4 & 48,4 \\
Concordo totalmente & 8 & 25,8 & 25,8 & 74,2 \\
Discordo & 8 & 25,8 & 25,8 & 100,0 \\
Total & $\mathbf{3 1}$ & $\mathbf{1 0 0 , 0}$ & $\mathbf{1 0 0 , 0}$ & \\
\hline
\end{tabular}

Fonte: Pesquisa direta (2018).

A maioria do alunado $(n=23$ ou 77,2\%) reconheceu que houve a indução a participar das discussões via fórum. Não obstante, um grupo minoritário ( $n=8$ ou 22,8\%) não reconheceu ter ocorrido indução à participação nas discussões via fórum. Essa é uma informação preocupante, que deve acionar novas estratégias por parte da equipe de gestão pedagógica do curso, pois a participação nas discussões via fórum é uma atividade vital ao desenvolvimento de novas competências e ao fortalecimento do aprendizado, conforme Moore e Kearsley (2008), Preti (2000) e Peters (2003).

Posteriormente averiguou-se a opinião do alunado acerca da indução a compartilhar ideias e conhecimentos através das várias metodologias de discussão, conforme revelam os dados da Tabela 21.

Tabela 21 - Os alunos são convidados a compartilhar suas ideias e conhecimentos

\begin{tabular}{ccccc}
\hline $\begin{array}{c}\text { CATEGORIAS DE } \\
\text { RESPOSTAS }\end{array}$ & FREQUÊNCIA & PORCENTUAL & $\begin{array}{c}\text { PORCENTAGEM } \\
\text { VÁLIDA }\end{array}$ & $\begin{array}{c}\text { PORCENTAGEM } \\
\text { ACUMULATIVA }\end{array}$ \\
\hline Concordo & 23 & 74,2 & 74,2 & 74,2 \\
Concordo totalmente & 8 & 25,8 & 25,8 & 100,0 \\
Total & 31 & 100,0 & 100,0 & \\
\hline
\end{tabular}

Fonte: Pesquisa direta (2018). 
A totalidade do alunado reconheceu que houve indução a compartilhar ideias e conhecimentos através das várias metodologias de discussão, empoderando-se, desse modo, o processo de formação online.

Em seguida avaliou-se o alunado acerca da indução e do encorajamento para responder as questões disponibilizadas no fórum, conforme os dados da Tabela 22.

Tabela 22 - Os alunos são encorajados a responder a questão central do fórum

\begin{tabular}{ccccc}
\hline $\begin{array}{c}\text { CATEGORIAS DE } \\
\text { RESPOSTAS }\end{array}$ & FREQUÊNCIA & PORCENTUAL & $\begin{array}{c}\text { PORCENTAGEM } \\
\text { VÁLIDA }\end{array}$ & $\begin{array}{c}\text { PORCENTAGEM } \\
\text { ACUMULATIVA }\end{array}$ \\
\hline Concordo & 21 & 67,7 & 67,7 & 67,7 \\
Concordo totalmente & 8 & 25,8 & 25,8 & 93,5 \\
Discordo & 2 & 6,5 & 6,5 & 100,0 \\
Total & 31 & $\mathbf{1 0 0 , 0}$ & $\mathbf{1 0 0 , 0}$ & \\
\hline
\end{tabular}

Fonte: Pesquisa direta (2018).

A expressiva maioria do alunado ( $n=29$ ou 93,5\%) reconheceu que houve indução e encorajamento para responder as questões disponibilizadas no fórum, empoderando, desse modo, o processo de debate e de formação online, conforme idealizado por Alonso (2010).

O alunado também foi sondado acerca de estímulos à proposição de questionamentos transversais aos temas e assuntos abordados no fórum, apresentados pelos tutores, conforme os dados da Tabela 23.

Tabela 23 - Os alunos são estimulados a propor questionamentos transversais ao tema central do fórum

\begin{tabular}{ccccc}
\hline $\begin{array}{c}\text { CATEGORIAS DE } \\
\text { RESPOSTAS }\end{array}$ & FREQUÊNCIA & PORCENTUAL & $\begin{array}{c}\text { PORCENTAGEM } \\
\text { VÁLIDA }\end{array}$ & $\begin{array}{c}\text { PORCENTAGEM } \\
\text { ACUMULATIVA }\end{array}$ \\
\hline Concordo & 20 & 64,5 & 64,5 & 64,5 \\
Concordo totalmente & 8 & 25,8 & 25,8 & 90,3 \\
Discordo & 3 & 9,7 & 9,7 & 100,0 \\
Total & 31 & $\mathbf{1 0 0 , 0}$ & $\mathbf{1 0 0 , 0}$ & \\
\hline
\end{tabular}

Fonte: Pesquisa direta (2018). 
Verifica-se que a expressiva maioria do alunado ( $n=28$ ou 90,3\%) reconheceu que houve estímulos à proposição de questionamentos transversais aos temas e assuntos centrais abordados no fórum, contribuindo para o adensamento da formação online.

Por fim, o alunado foi sondado acerca do interesse demonstrado pela equipe de tutores com o aprendizado, conforme revelam os dados da Tabela 24.

Tabela 24 - A equipe de tutores apresenta interesse pelo aprendizado do alunado

\begin{tabular}{|c|c|c|c|c|}
\hline $\begin{array}{c}\text { CATEGORIAS DE } \\
\text { RESPOSTAS }\end{array}$ & FREQUÊNCIA & PORCENTUAL & $\begin{array}{c}\text { PORCENTAGEM } \\
\text { VÁLIDA }\end{array}$ & $\begin{array}{l}\text { PORCENTAGEM } \\
\text { ACUMULATIVA }\end{array}$ \\
\hline Concordo & 19 & 61,3 & 61,3 & 61,3 \\
\hline Concordo totalmente & 10 & 32,3 & 32,3 & 93,5 \\
\hline Discordo & 1 & 3,2 & 3,2 & 96,8 \\
\hline Discordo totalmente & 1 & 3,2 & 3,2 & 100,0 \\
\hline Total & 31 & 100,0 & 100,0 & \\
\hline
\end{tabular}

Fonte: Pesquisa direta (2018).

Observa-se que a expressiva maioria do alunado ( $n=29$ ou 93,5\%) reconheceu a preocupação da equipe de tutores com o aprendizado, aspecto esse que contribui para o adensamento e o aquilatamento da formação online.

\section{Qualidade dos Processos Avaliativos}

Averiguar a qualidade dos processos avaliativos adotados pelos tutores é uma rotina que deve ser perseguida pelos gestores acadêmicos. Trata-se de estratégia de gestão que possibilita a geração de novas informações, garantindo-se a função diagnóstica da avaliação. Sob este prisma, os alunos foram indagados acerca da adequação das correções das várias atividades às quais são submetidos durante o processo formativo, conforme os dados da Tabela 25. 
Tabela 25 - Adequação das correções das avaliações do aprendizado

\begin{tabular}{ccccc}
\hline $\begin{array}{c}\text { CATEGORIAS DE } \\
\text { RESPOSTAS }\end{array}$ & FREQUÊNCIA & PORCENTUAL & $\begin{array}{c}\text { PORCENTAGEM } \\
\text { VÁLIDA }\end{array}$ & $\begin{array}{c}\text { PORCENTAGEM } \\
\text { ACUMULATIVA }\end{array}$ \\
\hline Concordo & 18 & 58,1 & 58,1 & 58,1 \\
Concordo totalmente & 7 & 22,6 & 22,6 & 80,6 \\
Discordo & 2 & 6,5 & 6,5 & 87,1 \\
Discordo totalmente & 4 & 12,9 & 12,9 & 100,0 \\
Total & $\mathbf{3 1}$ & $\mathbf{1 0 0 , 0}$ & $\mathbf{1 0 0 , 0}$ & \\
\hline
\end{tabular}

Fonte: Pesquisa direta (2018).

Verifica-se que a expressiva maioria do alunado ( $n=25$ ou 80,6\%) reconheceu a adequação dos procedimentos de correção das avaliações e dos trabalhos aos quais são submetidos. No entanto, grupo minoritário expressou sua discordância da opinião majoritária ( $\mathrm{n}=6$ ou 19,5\%).

Em seguida os alunos foram indagados acerca da justiça com que são executadas as avaliações às quais foram submetidos durante a formação, conforme a Tabela 26.

Tabela 26 - Justiça das avaliações do aprendizado

\begin{tabular}{ccccc}
\hline $\begin{array}{c}\text { CATEGORIAS DE } \\
\text { RESPOSTAS }\end{array}$ & FREQUÊNCIA & PORCENTUAL & $\begin{array}{c}\text { PORCENTAGEM } \\
\text { VÁLIDA }\end{array}$ & $\begin{array}{c}\text { PORCENTAGEM } \\
\text { ACUMULATIVA }\end{array}$ \\
\hline Concordo & 23 & 74,2 & 74,2 & 74,2 \\
Concordo totalmente & 1 & 3,2 & 3,2 & 77,4 \\
Discordo & 3 & 9,7 & 9,7 & 87,1 \\
Discordo totalmente & 4 & 12,9 & 12,9 & 100,0 \\
Total & 31 & $\mathbf{1 0 0 , 0}$ & $\mathbf{1 0 0 , 0}$ & \\
\hline
\end{tabular}

Fonte: Pesquisa direta (2018).

Observa-se que a expressiva maioria do alunado $(n=24$ ou 77,4\%) reconheceu a justiça dos procedimentos de avaliação aos quais foram submetidos durante a formação. No entanto, grupo minoritário expressou sua discordância da maioria ( $n=7$ ou 22,6\%).

Para finalizar, os alunos foram indagados acerca da abordagem dos conteúdos que foram avaliados pelos tutores durante o processo formativo, conforme a Tabela 27. 
Tabela 27 - Aderência das avaliações aos conteúdos efetivamente abordados nas aulas

\begin{tabular}{ccccc}
\hline $\begin{array}{c}\text { CATEGORIAS DE } \\
\text { RESPOSTAS }\end{array}$ & FREQUÊNCIA & PORCENTUAL & $\begin{array}{c}\text { PORCENTAGEM } \\
\text { VÁLIDA }\end{array}$ & $\begin{array}{c}\text { PORCENTAGEM } \\
\text { ACUMULATIVA }\end{array}$ \\
\hline Concordo & 21 & 67,7 & 67,7 & 67,7 \\
Concordo totalmente & 4 & 12,9 & 12,9 & 80,6 \\
Discordo & 2 & 6,5 & 6,5 & 87,1 \\
Discordo totalmente & 4 & 12,9 & 12,9 & 100,0 \\
Total & $\mathbf{3 1}$ & $\mathbf{1 0 0 , 0}$ & $\mathbf{1 0 0 , 0}$ & \\
\hline
\end{tabular}

Fonte: Pesquisa direta (2018).

Identifica-se que a expressiva maioria do alunado reconheceu que os conteúdos avaliados foram de fato abordados em sala de aula ( $n=25$ ou 80,6\%). Não obstante, um grupo minoritário expressou sua discordância ( $n=6$ ou 19,4\%).

\section{CONSIDERAÇÕES FINAIS}

Os desafios resultantes da expansão da Educação Superior no Brasil passam, inevitavelmente, pela discussão da qualidade das Instituições de Ensino Superior (IES) e as formas de avaliação, consoante Andriola e Suliano (2015), Araújo, Andriola e Coelho (2018), bem como pelo enfrentamento de dilemas resultantes da oferta de EaD, conforme Alonso (2010), Andriola e Loureiro (2005).

Para Marsh (1987), Marsch e Bailey (1993), discutir a qualidade da formação brindada por cursos de EaD deveria fazer-se a partir da valoração de cinco indicadores: (i) a qualidade do tutor online; (ii) a qualidade da interação tutor online com os alunos; (iii) a qualidade da aprendizagem dos alunos; (iv) a qualidade da relação tutor presencial com os alunos; (v) a qualidade dos processos avaliativos. Com base nesses elementos poder-se-á valorar a qualidade de cursos EaD.

Sob esta ótica, o presente estudo conseguiu avaliar a qualidade do curso de Pedagogia online ofertado pelo UNIGrande, a partir da opinião dos alunos sobre os indicadores propostos 
por Marsh (1987), Marsch e Bailey (1993). Os resultados fornecem elementos que atestam a elevada qualidade do curso, nos aspectos detalhados abaixo.

a) Perfil do curso: a maioria do alunado reconheceu o curso como desafiador e estimulante, com conteúdos pertinentes, motivadores e aderentes aos objetivos pretendidos pela formação, cujos recursos disponibilizados através do AVA são acessíveis e de fácil uso;

b) Qualidade do tutor online: a maioria dos alunos assegurou ter entusiasmo, amizade, estímulo ao aprofundamento de informações e ao incremento do aprendizado através de pesquisas em sites e outros espaços acadêmicos;

c) Qualidade da interação do tutor online: os alunos asseveraram que o tutor detinha conhecimentos adequados e compatíveis com os temas abordados, esclareceu dúvidas e incentivou a participação ativa nos fóruns de discussões no AVA, de modo a interagir e compartilhar ideias;

d) Qualidade da interação do tutor presencial: a maioria do alunado reconheceu que os tutores têm conhecimentos técnicos, habilidades e atitudes favoráveis ao aprendizado;

e) Qualidade dos materiais pedagógicos: o alunado opinou positivamente acerca a qualidade do material pedagógico usado na formação, com elogios ao uso textos;

f) Qualidade da aprendizagem: a maioria do alunado reconheceu a indução à participação ativa dos fóruns de discussões, de modo a interagir e compartilhar ideias;

g) Qualidade dos processos avaliativos: a maioria dos alunos atribuiu justiça e aderência das avaliações aos conteúdos abordados no processo de formação.

Portanto, pode-se inferir que o curso de Pedagogia online do UNIGrande conta com o amplo reconhecimento da comunidade discente no que tange aos critérios delimitadores da qualidade acadêmica propostos por H. W. Marsh. Para concluir e realçar o poder transformador da Educação, lembramos sábia frase proferida pelo líder negro norteamericano, Al Hajj Malik Al-Shabazz, mais conhecido pelo pseudônimo Malcom X: a Educação é o nosso passaporte para o futuro. 


\section{REFERÊNCIAS}

ALONSO, Kátia Morosov. A expansão do Ensino Superior no Brasil e a EaD: dinâmica e lugares. Educação e Sociedade, Campinas, v. 31, n. 113, p. 1319-1335, 2010. Disponível em: <http://www.scielo.br/scielo.php?script=sci_arttext\&pid=S0101-73302010000400014\&lng=en\&nrm=iso>. Acesso em 31/05/2019.

ANDRIOLA, Wagner Bandeira. Evaluación: la vía para la calidad educativa. Ensaio. Avaliação e Políticas Públicas em Educação, Rio de Janeiro, v. 7, n. 25, p. 355-368, 1999.

ANDRIOLA, Wagner Bandeira. Factores caracterizadores de centros educativos eficaces. Bordón: Revista de Pedagogía, Madrid, v. 53, n. 2, p. 175-183, 2001.

ANDRIOLA, Wagner Bandeira; Mc DONALD, Brendan Coleman (Org.). Avaliação: Fiat Lux em Educação. Fortaleza: Editora da Universidade Federal do Ceará, 2003.

ANDRIOLA, Wagner Bandeira. Avaliação institucional na Universidade Federal do Ceará (UFC): organização de sistema de dados e indicadores da qualidade institucional. Avaliação: Revista da Avaliação da Educação Superior, Campinas, v. 9, n.4, p. 33-54, 2004. Disponível em <http://periodicos.uniso.br/ojs/index.php/avaliacao/article/view/1286>. Acesso em 30/06/2019.

ANDRIOLA, Wagner Bandeira (Org.). Avaliação: múltiplos olhares em torno da educação. Fortaleza: Editora da Universidade Federal do Ceará, 2005a.

ANDRIOLA, Wagner Bandeira. Desafios e necessidades que se apresentam às Comissões Próprias de Avaliação (CPAs) das instituições de educação superior (IES), visando à implementação do Sistema Nacional de Avaliação da Educação Superior (SINAES) - (p. 57-70). In: RISTOFF, D. I.; ALMEIDA JÚNIOR, V. (Org.). Avaliação participativa: perspectives e debates. Brasília, DF: INEP, 2005b.

ANDRIOLA, Wagner Bandeira. Propostas estatais voltadas à avaliação do Ensino Superior brasileiro: Breve retrospectiva histórica do período 1983-2008. Revista Electrónica Iberoamericana Sobre Calidad, Eficacia y Cambio en Educación, Madrid, v. 6, p. 127-148, 2008a. Disponível em <http://www.rinace.net/reice/numeros/arts/vol6num4/art7.pdf>. Acesso em 24/05/2019.

ANDRIOLA, Wagner Bandeira; ANDRIOLA, Cristiany Gomes. Avaliação da qualidade educacional da Faculdade de Educação (FACED) da Universidade Federal do Ceará (UFC). Ensaio: Avaliação e Políticas Públicas em Educação, Rio de Janeiro, v. 17, n. 62, p. 153-168, 2009. Disponível em <http://www.scielo.br/pdf/ensaio/v17n62/a08v1762.pdf >. Acesso em 04/04/2019.

ANDRIOLA, Wagner Bandeira; LOUREIRO, Robson. Sistematização da avaliação da aprendizagem em comunidades organizadas no ciberespaço. Revista Iberoamericana de Educación, v. 37, n. 1, p. 1-6, 2005. Disponível em <https://rieoei.org/RIE/article/view/2729>. Acesso em 08/04/2019.

ARAUJO, Adriana Castro; ANDRIOLA, Wagner Bandeira; COELHO, Afrânio de Araújo. Programa Institucional de Bolsa de Iniciação à Docência (PIBID): desempenho de bolsistas versus não bolsistas. Educação em Revista, Belo Horizonte, $\quad$ v.34, $\quad$ e172839, $2018 . \quad$ Disponível el <http://www.scielo.br/scielo.php?script=sci_arttext\&pid=S0102-46982018000100112\&lng=en\&nrm=iso>. Acesso em 12/11/2018. 
ANDRIOLA, Wagner Bandeira; SULIANO, Daniele Cirilo. Avaliação dos impactos sociais oriundos da interiorização da Universidade Federal do Ceará (UFC). Revista Brasileira de Estudos Pedagógicos, Brasília, v.96, n.243, p.282-298, 2015. Disponível em $<$ http://www.scielo.br/scielo.php?script=sci_arttext\&pid=S2176-66812015000200282\&lng=en\&nrm=iso>. Acesso em 08/08/2018.

ALBUQUERQUE, Michele Rodrigues de; SILVA, Ivanda Maria Martins. Materiais didáticos impressos para Educação a Distância: interfaces com práticas de linguagem. EDT - Educação Temática Digital, Campinas, v.14, n.2, p.75-93, 2012.

BASTOS, Carmen Célia Barradas Correia; ROVARIS, Nelci Aparecida. A relevância do processo de autoavaliação institucional da universidade tecnológica para configuração do bom professor. Revista da Avaliação da Educação Superior, Campinas, v.21, n. 3, p. 767-781, 2016. Disponível em $<$ http://www.scielo.br/scielo.php?script=sci_arttext\&pid=S1414-40772016000300767\&lng=en\&nrm=iso>. Acesso em 24/10/2018.

BLEICHER, Sabrina. Processos flexíveis para a produção de materiais didáticos para a Educação a Distância: recomendações pautadas na perspectiva interdisciplinar. Tese de Doutorado em Engenharia e Gestão do Conhecimento (386 pág.). Universidade Federal de Santa Catarina (UFSC). Florianópolis, 2015.

BALZÁN, Newton César. O conceito de planejamento e sua aplicação aos sistemas educacionais e às atividades de ensino - alcance e limites no limiar do século XXI. Educação Brasileira, Brasília, v. 18, n. 37, p. 151-172, 1996.

BRASIL, Lei no 10.861, de 14 de abril de 2004. Institui o Sistema Nacional de Avaliação da Educação Superior SINAES e dá outras providências. Brasília, DF, 2004. Disponível em: $<$ http://www.planalto.gov.br/ccivil_03/_ato2004-2006/2004/lei/l10.861.htm>. Acesso em 28/12/2018.

CASAGRANDE, Lucas; KLERING, Luís Roque; KRUEL, Alexandra. Estudo comparativo de percepções de alunos de especialização lato sensu nas modalidades presencial e EaD. Encontro da Associação Nacional de PósGraduação e Pesquisa em Administração. Rio de Janeiro: Anais da ANPAD, 2008.

EATON, Judith. Maintaining the delicate balance: distance learning, higher education accreditation, and the politics of self-regulation. American Council on Education, Washington, 2002.

GARRIDSON, Randy. Quality and access in distance education: theoretical considerations (p. 313-324). In: KEEGAN, D (Ed.). Theoretical principles of distance education. London: Routledge, 1993.

HOPE, Andrea. Quality Assurance. In: FARRELL, G. (ed.). The Changing Face of Virtual Education. Vancouver: Commonwealth of Learning, 2001.

JOHNSON, Gaye; GLASMAN, Naftaly. Evaluation authority and financial control. Studies in Educational Evaluation, Washington, DC, v. 9, n. 1, p. 59-76, 1983.

LAVOR, João Ferreira; ANDRIOLA, Wagner Bandeira; LIMA, Alberto Sampaio. Avaliando o Impacto da Qualidade da Gestão Acadêmica no Desempenho dos Cursos de Graduação. Um Estudo em Universidade Pública Brasileira. Revista Iberoamericana de Evaluación Educativa, Santiago de Chile, v. 8, n. 2, p. 233-254, 2015. Disponível em $<$ http://www.redalyc.org/pdf/551/55125665008.pdf>. Acesso em 12/10/2018.

LIMA, Alberto Sampaio; ANDRIOLA, Wagner Andriola. Avaliação de prática pedagógicas inovadoras em cursos de graduação em Sistemas de Informação. Revista Iberoamericana sobre Calidad, Eficacia y Cambio em la 
Educación, Madrid, v. 11, n. 1, p. 104-121, 2013. Disponível em $<$ https://www.redalyc.org/pdf/643/64340413015.pdf>. Acesso em 12/10/2018.

LIMA, Leonardo Araújo; ANDRIOLA, Wagner Andriola. Acompanhamento de egressos: subsídios para a avaliação de Instituições de Ensino Superior (IES). Revista da Avaliação da Educação Superior, Campinas, v. 23, n. 1, p. 104-125, 2018. Disponível em <http://www.scielo.br/scielo.php?script=sci_arttext\&pid=S1414-

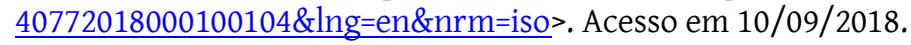

MAIA, Carmen; MATTAR, João. ABC da EaD: a educação a distância hoje. São Paulo: Pearson Prentice Hall, 2007.

MARSH, Herbert. W. Students' Evaluations of University Teaching: Research Findings, Methodological Issues and Directions for Future Research. International Journal of Educational Research, vol. 11, p. 253-388, 1987. Disponível em <https://eric.ed.gov/?id=ED338629>. Acesso em 26/03/2019.

MARSH, Herbert; BAILEY, Michael. Multidimensional students' evaluations of teaching effectiveness: A profile analysis. The Journal of Higher Education, v. 64, p. 1-18, 1993. Disponível em $<$ https://www.jstor.org/stable/2959975?seq=1\#page_scan_tab_contents>. Acesso em 31/05/2019.

MOORE, Michael. Editorial: Distance Education Theory. The American Journal of Distance Education, Pennsylvania, v. 5, n. 3, p. 1-6, 1991.

MCKINNON, Keneth Richards; WALKER, Suzanne; DAVIS, Dorothy. Benchmarking: A Manual for Australian Universities. Austrália: 2000.

MOORE, Michael; KEARSLEY, Greg. Educação a Distância: uma visão integrada. São Paulo: Cengage Learning, 2008.

NUNES, Albano Oliveira; SILVA, Thomaz Edson; MOTA, João César; ALMEIDA, André Férrer; ANDRIOLA, Wagner Bandeira. Developing an Instrument for Assessment of Academic Management in Engineering Courses. IEEE Latin América Transactions, Bogotá, v. 13, n. 1, p. 264-271, 2015a. Disponível em $<$ http://www.redalyc.org/pdf/643/64340413015.pdf>. Acesso em 29/10/2018.

NUNES, Albano Oliveira; SILVA, Thomaz Edson; MOTA, João César; ALMEIDA, André Férrer; ANDRIOLA, Wagner Bandeira. Validation of the academic management evaluation instrument based on principal component analysis for engineering and technological courses. Ingeniería e Investigación, Bogotá, v. 35, n. 2, p. 97-102, 2015b. Disponível em <http://www.scielo.org.co/scielo.php?script=sci_arttext\&pid=S0120$\underline{56092015000200015 \& \operatorname{lng}=e n \& n r m=i s o}>$. Acesso em 14/10/2018.

PEREIRA, Adriano Neves; BATISTA, Keila Moreira; DUARTE, Francisco Ricardo; BESERRA, Patrícia de Fátima Costa. Perfil dos Egressos em Formação Pedagógica a Distância em Ciência Biológicas da Universidade Federal do Vale do São Francisco. Anais do Simpósio Internacional de Educação à Distância. São Carlos - SP, 2014.

PETERS, Otto. A educação a distância em transição: tendências e desafios. São Leopoldo, UNISINOS, 2003.

PHIPPS, Ronald; MERISOTIS, Jamie. Quality on the Line - benchmarks for success in Internet-based distance education. Technical Report (42 p.). Washington, DC: The Institute for Higher Education Policy, 2000.

PRETI, Oreste. Autonomia do aprendiz na educação a distância (p. 125-146). In: PRETI, O. (Org.). Educação a distância: construindo significados. Cuiabá: NEAD/IE-UFMT; Brasília, DF: Plano, 2000. 
RAPCHAN, Francisco; CURY, Davidson; MENEZES, Crediné; FALBO, Ricardo de Almeida. EduQNet: Um Modelo de Qualidade de Processo para Cursos a Distância Mediados pela Internet. Simpósio Brasileiro de Qualidade de Software. Gramado, 2002.

RIBEIRO, Germano de Oliveira; NUNES, Albano Oliveira; COSTA, Francisco Ernandes Matos; SILVA; Thomaz Edsono Veloso; ANDRIOLA, Wagner Bandeira. Avaliação da eficácia do ensino em cursos de graduação a distância. Revista SUSTINERE, Rio de Janeiro, v. 6, n. 2, p. 222-238, 2018. Disponível em <https://www.epublicacoes.uerj.br/index.php/sustinere/article/view/36745>. Acesso em 05/09/2019.

SALES, Gilvandenys Leite; BARROSO, Giovanni Cordeiro; SOARES, José Marques; ANDRIOLA, Wagner Bandeira; JOYE, Cassandra Ribeiro. Um indicador de aprendizagem não-linear para EaD online fundamentado no modelo de avaliação Learning Vectors (LV). Revista Iberoamericana de Evaluación Educativa, v. 4, n. 2, p. 155-180, 2011. Disponível em <https://dialnet.unirioja.es/servlet/articulo?codigo=4522626>. Acesso em 01/07/2019.

SANTORO TRIGUEIRO, Michelangelo Giotto. A avaliação institucional e a redefinição das estruturas e modelos de gestão das Instituições de Ensino Superior do país. Revista da Rede de Avaliação Institucional da Educação Superior, Campinas, v. 9, n. 3, p. 11- 30, 2004. Disponível em <http://periodicos.uniso.br/ojs/index.php/avaliacao/article/view/1275>. Acesso em 01/07/2019.

SILVA, Francisco César Martins da; LIMA, Alberto Sampaio; ANDRIOLA, Wagner Bandeira. REICE. Avaliação do Suporte de TDIC na Formação do Pedagogo. Um Estudo em Universidade Brasileira. Revista Iberoamericana sobre Calidad, Eficacia y Cambio en Educación, Madrid, v. 14, n. 3, p. 77-93, 2016. Disponível em <http://www.redalyc.org/pdf/551/55146042004.pdf>. Acesso em 01/07/2019.

SILVA, Thomaz Edson Veloso; RIBEIRO, Germano Oliveira; NUNES, Albano Oliveira; VASCONCELOS, Francisco Herbert; ANDRIOLA, Wagner Bandeira; MOTA, João César Moura. QEOn Questionnaire for Assessing Experiences in Virtual Learning Environments. IEEE Latin America Transaction, v. 15, p. 1197-1204, 2017. Disponível em <https://www.researchgate.net/publication/316254079_QEOn_Questionnaire_for_Assessing_Experiences_in_ Virtual_Learning_Environments>. Acesso em 08/07/2019.

SILVA, Thomaz Edson Veloso; RIBEIRO, Germano Oliveira; NUNES, Albano Oliveira; VASCONCELOS, Francisco Herbert; ANDRIOLA, Wagner Bandeira. Avaliação dos indicadores da qualidade do ensino online: um estudo de caso. In: Anais do IV Congresso Brasileiro de Informática na Educação (CBIE 2015) e VIII Workshop sobre Avaliação e Acompanhamento da Aprendizagem em Ambientes Virtuais (Wavalia 2015), p. 503-511, 2015.

TEIXEIRA, Dirceu; RIBEIRO, Luiz dos Santos; CASSIANO, Keila Mara; MASUDA, Oya; BENCHIMOL, Marlene. Perfil e Destino Ocupacional de Egressos Graduados em Ciências Biológicas nas Modalidades a Distância e Presencial. Ensaio Pesquisa em Educação em Ciências, Belo Horizonte, v.16, n.1, p. 67-84, 2014.

TOLENTINO, Renata de Sousa da Silva; GONÇALVES FILHO, Cid; TOLENTINO, Ricardo José Vaz; MONTEIRO, Plínio Rafael Reis. Avaliação da qualidade na Educação a Distância sob a perspectiva do aluno: proposição e teste de um modelo usando equações estruturais. Revista de Gestão, v. 20, n. 3, p. 347-366, 2013.

(cc) BY

Este trabalho está licenciado com uma Licença Creative Commons - Atribuição 4.0 Internacional. 